\title{
MORE ON THE CONTINUITY OF THE REAL ROOTS OF AN ALGEBRAIC EQUATION
}

\author{
J. R. ISBELL
}

Melvin Henriksen and I published [1] an incomplete restoration of the following theorem announced by Hewitt [2]:

Theorem. Let $C(X, R)$ be the ring of all continuous real-valued functions on a completely regular space $X$; let $M$ be a maximal ideal in $C(X, R)$. The residue field $C(X, R) / M=C_{M}$ is real closed.

Hewitt's proof is defective only in showing a root for every polynomial of odd degree in $C_{M}$; we used other results of [2] and the Tietze extension theorem, i.e., we proved the theorem for normal $X$. This note recovers the whole theorem.

Proof of Theorem. After Hewitt's work [2], it remains to show that every polynomial $P(x, w)=w^{2 n+1}+\sum_{k=0}^{2 n} a_{k}(x) w^{k}, a_{k} \in C(X, R)$, has a root in $C_{M}$. If $f \in C(X, R)$, let $Z(\tilde{f})=[x \in X \mid f(x)=0], Z(M)$ $=[Z(f) \mid f \in M]$. Decompose the real part of the root of $P$ into continuous single-valued functions, $\phi_{1}, \cdots, \phi_{2 n+1}$, as in [1]. Let $R_{i}$ $=\left[x \in X \mid P\left(x, \phi_{i}(x)\right)=0\right]$. Since the $R_{i}$ cover $X$ and $Z(M)$ has the finite intersection property, some $R_{i *}$ meets every element of $Z(M)$. Then by $[2$, Theorem 36$], R_{i *} \in Z(M)$; that is, $P\left(x, \phi_{i *}(x)\right) \equiv 0$ $(\bmod M)$.

\section{REFERENCES}

1. M. Henriksen and J. R. Isbell, On the continuity of the real roots of an algebraic equation, Proc. Amer. Math. Soc. vol. 4 (1953) pp. 431-434.

2. E. Hewitt, Rings of real-valued continuous functions. I, Trans. Amer. Math. Soc. vol. 64 (1948) pp. 45-99.

Princeton University

Presented to the Society, October 24, 1953; received by the editors September 21. 1953. 